NDA-FP-53

December 1998

\title{
Two-Boundaries AdS/CFT Correspondence in Dilatonic Gravity
}

\author{
SHIN'ICHI NOJIRI II and SERGEI D. ODINTSOV P \\ Department of Mathematics and Physics \\ National Defence Academy, Hashirimizu Yokosuka 239, JAPAN \\ A Tomsk Pedagogical University, 634041 Tomsk, RUSSIA
}

\begin{abstract}
We discuss dilatonic gravity (bulk theory) from the point of view of (generalized) AdS/CFT correspondence. Self-consistent dilatonic background is considered. It may be understood as two boundaries space where AdS boundary appears as infinite boundary and new (singular) boundary occurs at short distances. The two-point correlation function and conformal dimension for minimal and dilaton coupled scalar are found. Even for minimal scalar, the conformal dimension is found to be different on above two boundaries. Hence, new CFT appears in AdS/CFT correspondence at short distances. AdS/CFT correspondence may be understood as interpolating bulk theory between two different CFTs.
\end{abstract}

\footnotetext{
${ }^{1}$ e-mail: nojiri@cc.nda.ac.jp

2 e-mail: odintsov@mail.tomsknet.ru
} 
AdS/CFT correspondence [1, 22, 3] gives an interesting framework to relate (classical) bulk theory with the conformal field theory living on the infinite boundary. In the original version of AdS/CFT corespondence, it has only one boundary (AdS). However, there are some indications [4] that singularities which appear and in bulk and in boundary theories could mean the opening of a new space. For example, it happens [4] that singular branes become regular in a dual, conformally rescaled, frame. That may indicate that there naturally appears second boundary (vacuum) in AdS/CFT correspndence [ [4, 5]. In other words, brane probably should not reside only at the infinite boundary of AdS [5] and it is better to imagine the brane is everywhere.

In the present letter, we discuss AdS/CFT correspondence for dilatonic theories in the space with two boundaries. We concentrate on the behaviour of correlation function of scalar field in different cases (minimal scalars, dilaton coupled scalars (see [7] for introduction)) on new short distance boundary. It is shown that CFT on this boundary is different from the one on infinite AdS boundary. The shift of conformal dimension due to mass and curvature coupling is also calculated. Finally, we give some remarks about another representation of dilatonic gravity under discussion as higher derivative gravity.

We start from the following action of dilatonic gravity in $d+1$ dimensions:

$$
S=-\int d^{d+1} x \sqrt{-g}\left(R-\Lambda-\alpha g^{\mu \nu} \partial_{\mu} \phi \partial_{\nu} \phi\right)
$$

In the following, we assume $\lambda^{2} \equiv-\Lambda$ and $\alpha$ to be positive. From the variation of the metric $g^{\mu \nu}$, we obtain[

$$
0=R_{\mu \nu}-\frac{1}{2} g_{\mu \nu} R+\frac{\Lambda}{2} g_{\mu \nu}+\alpha\left(\partial_{\mu} \phi \partial_{\nu} \phi-\frac{1}{2} g_{\mu \nu} g^{\rho \sigma} \partial_{\rho} \phi \partial_{\sigma} \phi\right)
$$

and from that of dilaton $\phi$

$$
0=\partial_{\mu}\left(\sqrt{-g} g^{\mu \nu} \partial_{\nu} \phi\right)
$$

3 The conventions of curvatures are given by

$$
\begin{aligned}
R & =g^{\mu \nu} R_{\mu \nu} \\
R_{\mu \nu} & =-\Gamma_{\mu \lambda, \kappa}^{\lambda}+\Gamma_{\mu \kappa, \lambda}^{\lambda}-\Gamma_{\mu \lambda}^{\eta} \Gamma_{\kappa \eta}^{\lambda}+\Gamma_{\mu \kappa}^{\eta} \Gamma_{\lambda \eta}^{\lambda} \\
\Gamma_{\mu \lambda}^{\eta} & =\frac{1}{2} g^{\eta \nu}\left(g_{\mu \nu, \lambda}+g_{\lambda \nu, \mu}-g_{\mu \lambda, \nu}\right) .
\end{aligned}
$$


We assume that solutions for $g_{\mu \nu}$ and $\phi$ depend only on one of the coordinate, say $y \equiv x^{d}$

$$
g_{\mu \nu}=g_{\mu \nu}(y), \phi=\phi(y)
$$

and $g_{\mu \nu}$ has the following form

$$
d s^{2}=\sum_{\mu, \nu=0}^{d} g_{\mu \nu} d x^{\mu} d x^{\nu}=f(y) d y^{2}+g(y) \sum_{i, j=0}^{d-1} \eta_{i j} d x^{i} d x^{j}
$$

Here $\eta_{i j}$ is the metric in the flat (Lorentzian) background. Then the equations of motion (2) and (3) take the following forms:

$$
\begin{aligned}
& 0=-\frac{d(d-1)}{8}\left(\frac{g^{\prime}}{g}\right)^{2}+\frac{\lambda^{2}}{2} f+\frac{\alpha}{2}\left(\phi^{\prime}\right)^{2} \\
& 0=-\frac{d-1}{2} \frac{g^{\prime \prime}}{g}+\frac{d-1}{4} \frac{f^{\prime} g^{\prime}}{f g}-\frac{(d-1)(d-4)}{8}\left(\frac{g^{\prime}}{g}\right)^{2}+\frac{\lambda^{2}}{2} f-\frac{\alpha}{2}\left(\phi^{\prime}\right)^{2}(7) \\
& 0=\left(\sqrt{\frac{g^{d}}{f}} \phi^{\prime}\right)^{\prime}
\end{aligned}
$$

Here $^{\prime}$ expresses the derivative with respect to $y$. Eq.(6) corresponds to $(\mu, \nu)=(d, d)$ in (2) and Eq.(17) to $(\mu, \nu)=(i, j)$. The case of $(\mu, \nu)=(0, i)$ or $(i, 0)$ is identically satisfied. Integrating (8), we find

$$
\phi^{\prime}=c \sqrt{\frac{f}{g^{d}}} .
$$

Substituting (9) into (6), we can solve it algebraically with respect to $f$ :

$$
f=\frac{d(d-1)\left(g^{\prime}\right)^{2}}{4 g^{2}\left(\lambda^{2}+\frac{\alpha c^{2}}{g^{d}}\right)}
$$

We find that Eq.(7) is automatically satisfied when we substitute (9) and (10). Therefore $g$ can be an arbitrary function of $y$ but this corresponds to the degree of the freedom of the reparametrization of $y$ in the metric (5). We can fix it by choosing

$$
g=y
$$


Then we find from (9) and (10),

$$
\begin{aligned}
f & =\frac{d(d-1)}{4 y^{2}\left(\lambda^{2}+\frac{\alpha c^{2}}{y^{d}}\right)} \\
\phi & =c \int d y \sqrt{\frac{d(d-1)}{4 y^{d+2}\left(\lambda^{2}+\frac{\alpha c^{2}}{y^{d}}\right)}} \\
& =\phi_{0}+\frac{1}{2} \sqrt{\frac{(d-1)}{d \alpha}} \ln \left\{\frac{2 \alpha c^{2}}{\lambda^{2} y^{d}}+1 \pm \sqrt{\left(\frac{2 \alpha c^{2}}{\lambda^{2} y^{d}}+1\right)^{2}-1}\right\} .
\end{aligned}
$$

From Eq.(13), we find the dilaton field behaves as $\phi \rightarrow \mp \sqrt{\frac{d(d-1)}{\alpha}} \ln y^{d}$ when $y \rightarrow 0$. Since the string coupling constant $\mathrm{e}^{\phi}$ should be small in order that the supergravity picture is consistent, the dilaton field cannot grow up positively. Therefore the - sign should be chosen in the \pm sign in Eq.(13) and we find

$$
\phi \rightarrow \frac{1}{2} \sqrt{\frac{d(d-1)}{\alpha}} \ln y .
$$

The metric given by (5), (11) and (12) becomes that of the usual anti-de Sitter space in the limit where $\alpha$ or $c$ vanishes.

The backreaction from the non-trivial background of dilaton (13) to the metric as in (12) changes the structure of the spacetime near the region where $y=0$. When $y$ is small, the metric behaves as

$$
d s^{2} \sim \frac{d(d-1)}{4 \alpha c^{2}} y^{d-2} d y^{2}+y \sum_{i, j=0}^{d-1} \eta_{i j} d x^{i} d x^{j} .
$$

The metric tells that the distance $l$ between the point with finite $y=y_{0}$ and that of $y=0$ is finite:

$$
l=\int_{y=y_{0}}^{y=0} d s=\int_{0}^{y_{0}} d y\left(\frac{d(d-1)}{4 \alpha c^{2}}\right)^{\frac{1}{2}} y^{\frac{d-2}{2}}=\left(\frac{(d-1)}{4 d \alpha c^{2}}\right)^{\frac{1}{2}} y_{0}^{\frac{d}{2}} .
$$

This should be compared with the case of usual anti-de Sitter space where the distance is infinite. In the limit where $\alpha$ or $c$ vanishes, the distance $l$ in 
(16) becomes infinite as expected. It ahould be also noted that there is a curvature singularity at $y=0$ since the scalar curvature is given by

$$
R=-\frac{(d+1) \Lambda}{d-1}-\alpha c^{2} y^{-d} \sim \alpha c^{2} y^{-d} .
$$

The infinite boundary discussed in AdS/CFT correspondence lies at $y=$ $\infty$. When $y \rightarrow \infty, f$ and $\phi$ in the solution (12), (13) behave as

$$
f \rightarrow \frac{d(d-1)}{4 \lambda^{2} y^{2}}\left(1+\mathcal{O}\left(y^{-d}\right)\right), \quad \phi \rightarrow \phi_{0}\left(1+\mathcal{O}\left(y^{-\frac{d}{2}}\right)\right)
$$

Therefore the geometry of spacetime asymptotically approaches to that of AdS, which tells the correlation functions of matter fields on the boundary corresponding to $y=\infty$ do not change with those on the boundary of AdS.

After Wick rotating the spacetime signature by changing $x^{0} \rightarrow i x^{0}$, as an example, we consider free massless scalar whose action is given by

$$
S^{\chi}=\frac{1}{2} \int d^{d+1} x \sqrt{g} g^{\mu \nu} \partial_{\mu} \chi \partial_{\nu} \chi
$$

and consider the correlation function in the neighborhood of the boundary $y=\infty$ by solving the following equation

$$
\begin{aligned}
\sqrt{g} \square G\left(y, X^{2}\right) & \sim A_{0} \partial_{y}\left(y^{\frac{d}{2}+1} \partial_{y} G\left(y, X^{2}\right)\right)+\frac{y^{\frac{d}{2}-2}}{A_{0}} \sum_{i=0}^{d-1} \partial_{i}^{2} G\left(y, X^{2}\right) \\
& =0 \\
\sqrt{g} \square & \equiv \partial_{\mu}\left(\sqrt{g} g^{\mu \nu} \partial_{\nu}\right) \\
A_{0} & \equiv 2 \sqrt{\frac{\lambda^{2}}{d(d-1)}} \\
X^{2} & \equiv \sum_{i=0}^{d-1}\left(x_{1}^{i}-x_{2}^{i}\right)^{2} .
\end{aligned}
$$

The solution of above equation near the infinite boundary is given by

$$
G\left(y, X^{2}\right)=G_{0} y^{-\frac{d}{2}}\left\{\left(\frac{4}{A_{0}^{2}} y^{-1}+X^{2}\right)^{-d}+\mathcal{O}\left(y^{-d}\right)\right\} .
$$


Here $G_{0}$ is a constant. Equation (21) gives a correlation function at $y=\infty$ as in [2]. In the limit of $y \rightarrow \infty$, we obtain

$$
G\left(y=0, X^{2}\right)=G_{0} y^{-\frac{d}{2}}\left(X^{2}\right)^{-d} .
$$

This correlation function is nothing but that of the operators with the conformal dimension $d$ in some kind of conformal field theory.

Since there is a singularity at $y=0$, we need to check if the fields at the boundary $y=\infty$ have a unique extension in the bulk spacetime $y<\infty$. As an example, we consider free massless scalar in (19). Since $\sqrt{g}$ is given by

$$
\sqrt{g}=\sqrt{\frac{d(d-1) y^{d-2}}{4\left(\lambda^{2}+\frac{\alpha c^{2}}{y^{d}}\right)}},
$$

the square-integrability requires

$$
\begin{array}{ll}
\chi \sim o\left(y^{-\frac{d}{4}}\right) & \text { when } y \rightarrow \infty \\
\chi \sim o\left(y^{-\frac{d}{2}}\right) & \text { when } y \rightarrow 0 .
\end{array}
$$

If the Laplace equation $\square \chi=0$ has a square integrable solution whose boundary value at $y=\infty$ vanishes, the uniqueness is broken since we can add the solution to any given solution. The solution can be written using the Fourier transformation with respect to the coordinates $\left\{x^{i}, i=1, \cdots, d\right\}$ as follows:

$$
\chi\left(y, x^{i}\right)=\frac{1}{(2 \pi)^{\frac{d}{2}}} \int d^{d} k f_{k}(y) \mathrm{e}^{i \sum_{i=0}^{d-1} k_{i} x^{i}} .
$$

Then the Laplace equation is rewritten as

$$
\begin{aligned}
0 & =\sqrt{g} \square \chi \\
\rightarrow & \frac{2}{\sqrt{d(d-1)}} \partial_{y}\left(y^{\frac{d}{2}+1} \sqrt{\lambda^{2}+\frac{\alpha c^{2}}{y^{d}}} \partial_{y} f_{k}(y)\right) \\
& -\frac{\sqrt{d(d-1)}}{2} \frac{k^{2} y^{\frac{d}{2}-1}}{\sqrt{\lambda^{2}+\frac{\alpha c^{2}}{y^{d}}}} f_{k}(y)
\end{aligned}
$$


Multiplying $f_{k}^{*}(y)$ (the complex conjugate of $f_{k}(y)$ ) and integrating with respect to $y$, we obtain

$$
\begin{aligned}
0= & \int_{0}^{\infty} d y\left\{\frac{2 y^{\frac{d}{2}+1}}{\sqrt{d(d-1)}} \sqrt{\lambda^{2}+\frac{\alpha c^{2}}{y^{d}}}\left|\partial_{y} f_{k}(y)\right|^{2}+\frac{\sqrt{d(d-1)}}{2} \frac{k^{2} y^{\frac{d}{2}-1}}{\sqrt{\lambda^{2}+\frac{\alpha c^{2}}{y^{d}}}}\left|f_{k}(y)\right|^{2}\right\} \\
& -2 \sqrt{\left.\frac{\alpha c^{2}}{d(d-1)} y f_{k}^{*}(y) \partial_{y} f_{k}(y)\right|_{y \rightarrow 0}} .
\end{aligned}
$$

Here we assume Eq.(24) and that $\chi$ vanishes at the boundary $y=\infty$ $\left(f_{k}(\infty)=0\right)$. Eq. (27) tells that if $f_{k}(y)$ does not vanish at $y=0$, there can be non-trivial square-integrable solutions whose boundary value at $y=\infty$ vanishes. This situation is very different from that in the usual AdS and the boundary value $\chi\left(y=\infty, x^{i}\right)$ cannot uniquely determine the value of $\chi$ in the bulk $y<\infty$. Eq.(27) tells, however, $f_{k}(y)=\partial_{y} f_{k}(y)=0$ everywhere if $\chi$ vanishes at $y=0\left(f_{k}(0)=0\right)$ and there is no any non-trivial squareintegrable solution. Note that the square-integrability requires $\chi$ vanish at $y=\infty$ due to (24). This implies that the value $\chi\left(y=0, x^{i}\right)$ at $y=0$ can uniquely determine the value of $\chi$ in the bulk $y>0$. Since there is a curvature singularity at $y=0$ (17), $y=0$ can be regarded as a boundary, which is similar to the case of Schwarzschild spacetime although the singularity discussed here is naked From the metric in (5), the new boundary at $y=0$ has also the topology of $d$-dimensional Minkowski space in the Minkowski signature. In the usual AdS/CFT correspondence, the boundary at $y=\infty$ can be regarded as a brane in superstring or M-theory. Since the solution (13) tells that there is a source of dilaton at $y=0$, the object at $y=0$ could be considered as brane with a dilatonic hair. (For classification of non-singular branes see [6]). If it is so, it is not so unnatural to expect that some kind of conformal field theory is realized on the boundary at $y=0$ (like it happened in the example of 3d AdS gravity in ref. [5]).

We now consider the correlation function of free massless scalar whose

\footnotetext{
${ }^{4}$ More exactly, if $f(y) \sim \sqrt{-\ln y}$ when $y \sim 0$, the boundary term in 27) becomes finite. The more singular behaviour of $f_{k}(y)$ can be consistent with the conditon of the square integrability in (24). In such a case, the boundary term, and therefore the bulk integration, in (27) diverges.

${ }^{5}$ If $\alpha<0$, there is a horizon since $f(y)$ in (12) diverges at $y^{d}=-\frac{\alpha c^{2}}{\lambda^{2}}$. In this case, the singularity is not naked.
} 
action is given by (19). We only consider the neighborhood of the boundary $y \sim 0$ and solve the following equation for correlation function

$$
\begin{aligned}
\sqrt{g} \square G\left(y, X^{2}\right) & \sim A \partial_{y}\left(y \partial_{y} G\left(y, X^{2}\right)\right)+\frac{y^{d-2}}{A} \sum_{i=0}^{d-1} \partial_{i}^{2} G\left(y, X^{2}\right) \\
& =0 \\
\sqrt{g} \square & \equiv \partial_{\mu}\left(\sqrt{g} g^{\mu \nu} \partial_{\nu}\right) \\
A & \equiv 2 \sqrt{\frac{\alpha c^{2}}{d(d-1)}} \\
X^{2} & \equiv \sum_{i=0}^{d-1}\left(x_{1}^{i}-x_{2}^{i}\right)^{2} .
\end{aligned}
$$

The solution of $(28)$ near the boundary $y \sim \infty$ is given by

$$
G\left(y, X^{2}\right)=G_{0}\left(\frac{4}{(d-1)^{2} A^{2}} y^{d-1}+X^{2}\right)^{-\frac{d}{2}} .
$$

Here $G_{0}$ is a constant. Equation (29) would give a correlation function on the boundary [2]. In fact, in the limit of $y \rightarrow 0$, we obtain

$$
G\left(y=0, X^{2}\right)=G_{0}\left(X^{2}\right)^{-\frac{d}{2}} .
$$

The correlation function (30) (which is different from one on the infinite boundary) is nothing but that of the operators with the conformal dimension $\frac{d}{2}$ in some kind of conformal field theory. Especially when $d=2$, the correlation function is that of the product of left-moving and right-moving free fermions $\mathcal{O}\left(x^{1}, x^{2}\right) \equiv \psi(z) \psi^{*}\left(z^{*}\right)\left(z=x^{1}+i x^{2}\right)$.

As a more general case, we consider the correlation function of massless dilaton coupled scalar whose action is given by

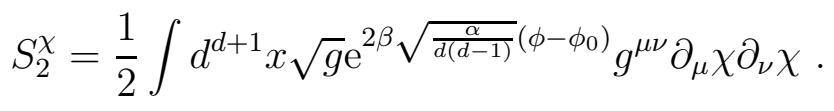

Here $\beta$ is a parameter which is now introduced. From (14), we find near the boundary

$$
\mathrm{e}^{2 \beta \sqrt{\frac{\alpha}{d(d-1)}}\left(\phi-\phi_{0}\right)} \sim y^{\beta} .
$$


Then in order to find the correlation function in the neighborhood of the boundary $y \sim 0$, we should solve the following equation instead of (28):

$$
\begin{aligned}
\sqrt{g} \square^{\phi} G^{\phi}\left(y, X^{2}\right) & \sim A \partial_{y}\left(y^{\beta+1} \partial_{y} G^{\phi}\left(y, X^{2}\right)\right)+\frac{y^{\beta+d-1}}{A} \sum_{i=0}^{d-1} \partial_{i}^{2} G^{\phi}\left(y, X^{2}\right) \\
& =0 \\
\sqrt{g} \square^{\phi} & \equiv \partial_{\mu}\left(\mathrm{e}^{2 \beta \sqrt{\frac{\alpha}{d(d-1)}}\left(\phi-\phi_{0}\right)} \sqrt{g} g^{\mu \nu} \partial_{\nu}\right) .
\end{aligned}
$$

The solution of (33) near the boundary $y \sim 0$ is given by

$$
G^{\phi}\left(y, X^{2}\right)=G_{0}\left(\frac{4}{(d-1)^{2} A^{2}} y^{d-1}+X^{2}\right)^{-\frac{d}{2}-\frac{\beta}{d-1}} .
$$

In the limit of $y \rightarrow 0$, we obtain

$$
G^{\phi}\left(y=0, X^{2}\right)=G_{0}\left(X^{2}\right)^{-\frac{d}{2}-\frac{\beta}{d-1}} .
$$

The correlation function (35) is that of the operators with the conformal dimension $\frac{d}{2}+\frac{\beta}{d-1}$. It is interesting that the conformal dimension is shifted by the parameter $\beta$ which comes from the dilaton coupling in (31). In the usual AdS/CFT correspondence, such a shift comes from the mass of the scalar field [2].

It would be also interesting to investigate the effect from the mass term. We add the following dilaton dependent mass term to the action (31):

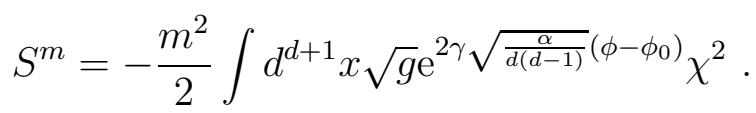

Then the equation coresponding to (28) is given by

$$
\begin{aligned}
& \sqrt{g}\left(\square^{\phi}-m^{2} \mathrm{e}^{2 \gamma \sqrt{\frac{\alpha}{d(d-1)}}\left(\phi-\phi_{0}\right)}\right) G^{m}\left(y, X^{2}\right) \\
& \sim A \partial_{y}\left(y^{\beta+1} \partial_{y} G^{m}\left(y, X^{2}\right)\right)+\frac{y^{\beta+d-1}}{A} \sum_{i=0}^{d-1} \partial_{i}^{2} G^{m}\left(y, X^{2}\right) \\
& -\frac{m^{2}}{A} y^{\gamma+d-3} G^{m}\left(y, X^{2}\right) \\
& =0
\end{aligned}
$$


In order that $G^{m}\left(y, X^{2}\right)$ corresponds to the correlation function of the conformal field theory on the boundary, $G^{m}\left(y, X^{2}\right)$ should have the following form in the limit of $y \rightarrow 0$ :

$$
G^{m} \sim \frac{y^{\rho}}{\left(X^{2}\right)^{\nu}} .
$$

Substituting (38) into (37), we find that $\gamma$ in (36) cannot be arbitrary but $\gamma$ should be given by

$$
\gamma=\beta-d+2 .
$$

When the equation (39) is satisfied, solution of (36) near the boundary $y \sim 0$ is given by

$$
G^{m}\left(y, X^{2}\right)=G_{0} y \frac{-\beta \pm \sqrt{\beta^{2}+\frac{4 m^{2}}{A^{2}}}}{2}\left(\frac{4}{(d-1)^{2} A^{2}} y^{d-1}+X^{2}\right)^{-\frac{d}{2} \mp \frac{\sqrt{\beta^{2}+\frac{4 m^{2}}{A^{2}}}}{d-1}} .
$$

In the limit of $y \rightarrow 0$, we obtain

$$
G^{m}\left(y=0, X^{2}\right)=G_{0} y^{\frac{-\beta \pm \sqrt{\beta^{2}+\frac{4 m^{2}}{A^{2}}}}{2}}\left(X^{2}\right)^{-\frac{d}{2} \mp \frac{\sqrt{\beta^{2}+\frac{4 m^{2}}{A^{2}}}}{d-1}} .
$$

The correlation function (41) is that of the operators with the conformal dimension $\frac{d}{2} \pm \frac{\sqrt{\beta^{2}+\frac{4 m^{2}}{A^{2}}}}{d-1}$.

We can also consider the coupling of the matter scalar field $\chi$ with the scalar curvature $R[7]$ :

$$
S^{R}=-\frac{\mu^{2}}{2} \int d^{d+1} x \sqrt{g} R \mathrm{e}^{2 \delta \sqrt{\frac{\alpha}{d(d-1)}}\left(\phi-\phi_{0}\right)} \chi^{2} .
$$

Near the boundary $y \sim 0$ the behavior of $R$ is given by (17). Comparing (17) with (32), we can identify near the boundary

$$
R \sim \alpha c^{2} \mathrm{e}^{-2 \sqrt{\frac{d \alpha}{(d-1)}}\left(\phi-\phi_{0}\right)} .
$$

Therefore by the following replacement,

$$
\gamma \rightarrow \delta-d, \quad m^{2} \rightarrow \mu^{2} \alpha c^{2}
$$


we can use the results in (39), (40) and (41).

Finally we show that the gravity theory in (1) can be rewritten as a higher derivative gravity theory without dilaton. When we rescale the metric by

$$
g_{\mu \nu} \rightarrow \mathrm{e}^{\rho} g_{\mu \nu}
$$

the action (1) is rewritten after the partial integration as follows

$$
S=-\int d^{d+1} x \sqrt{-g} \mathrm{e}^{\frac{d-1}{2} \rho}\left(R-\Lambda \mathrm{e}^{\rho}+\frac{(d-1) d}{4} g^{\mu \nu} \partial_{\mu} \rho \partial_{\nu} \rho-\alpha g^{\mu \nu} \partial_{\mu} \phi \partial_{\nu} \phi\right)
$$

Choosing $\rho$ as

$$
\rho=2 \sqrt{\frac{\alpha}{d(d-1)}} \phi
$$

we obtain

$$
S=-\int d^{d+1} x \sqrt{-g}\left\{\Phi^{d-1}\left(R-\Lambda \Phi^{2}\right)\right\}
$$

Here

$$
\Phi \equiv \mathrm{e}^{-\sqrt{\frac{\alpha}{d(d-1)}} \phi}
$$

By using the equation of motion with respect to $\Phi$, we can solve $\Phi$ with respect to the scalar curvature $R$ :

$$
\Phi^{2}=\frac{d-1}{(d+1) \Lambda} R
$$

Substituting (50) into (48), we obtain the higher derivative gravity theory which contains $\frac{d+1}{2}$-power of the scalar curvature $R$ :

$$
S=-\frac{2}{d+1}\left(-\frac{d-1}{(d+1) \Lambda}\right)^{d-1} \int d^{d+1} x \sqrt{-g}(-R)^{\frac{d+1}{2}} .
$$

In the usual AdS/CFT correspondence, we believe that the conformal symmetry on the boundary manifold comes from the $S O(d, 2)$ symmetry in the anti-de Sitter space but the spacetime given by (5), (11) and (12) has no the $S O(d, 2)$ symmetry. The obtained correlation functions (30), (35) and (41), however, seem to be those in some kind of conformal field theory. We should note that the action (51) is invariant under the global scale transformation with a constant parameter $c$

$$
g_{\mu \nu} \rightarrow \mathrm{e}^{c} g_{\mu \nu}
$$


which might be the origin of the conformal symmetry on the boundary.

In summary, we discussed CFT which appears on (short distance) $y=0$ boundary of dilatonic spacetime under consideration in generalized AdS/CFT correspondence. Dilatonic gravity may be considered as bulk theory interpolating between two different CFTs living at the boundaries $y=0$ and $y=\infty$. It could be really interesting to study AdS/CFT correspondence for the spacetimes with second boundary (of above sort) in $\mathrm{N}=4$ conformal supergravity [9, 10, 11] (more exactly gauged supergravity) where at $y=\infty$ boundary $\mathcal{N}=4$ super Yang-Mills theory lies [B] as dilaton naturally appears there.

Acknowledgements. We would like to acknowledge helpful discussions with T. Hollowood, P.van Nieuwenhuizen and E. Mottola. The work by SDO has been partially supported by T-8, LANL and RFBR project n96-02-16017.

\section{References}

[1] J.M. Maldacena, Adv.Theor.Math.Phys. 2 (1998) 231, hep-th/9711200.

[2] E. Witten, Adv.Theor.Math.Phys. 2 (1998) 505, hep-th/9802150.

[3] S. Gubser, I. Klebanov and A. Polyakov, Phys.Lett. B428 (1998) 105, hep-th/9802109.

[4] H.J. Boonstra, K. Skenderis and P.K. Townsend, hep-th/9807137.

[5] K. Behrndt, hep-th/9809015.

[6] G.W. Gibbons, G.T. Horowitz and P.K. Townsend, Class.Quant.Grav. 12 (1995) 287.

[7] S. Nojiri and S.D. Odintsov, Phys.Rev. D57 (1998) 2363.

[8] H. Liu and A.A. Tseytlin, preprint hep-th/9804083.

[9] M. Kaku, P.K. Townsend and P. van Nieuwenhuisen, Phys.Rev. D17 (1978) 3179.

[10] E. Bergshoeff, M. de Roo and B. de Wit, Nucl.Phys. B182 (1981) 233. 
[11] E.S. Fradkin and A.A. Tseytlin, Phys.Repts. 119 (1985) 233. 